\title{
¿Puede el principio de lateralidades múltiples mejorar el porcentaje de acierto en el tiro a canasta?
}

\section{May the multiple dominances principle improve the percentage average in the basketball shot?}

\author{
¿Pode o princípio da lateralidades várias melhorar a taxa de \\ sucesso no arremesso para uma cesta de campo? \\ C.J. Lopez-Diaz', N. Niño García ${ }^{2}$, M. Sillero Quintana ${ }^{3}$ y A. Lorenzo Calvo \\ ${ }^{1}$ Club Estudiantes; Universidad Politécnica de Madrid. ${ }^{2}$ Villaviciosa de Odón A.B. $y^{3}$ Universidad Politécnica de Madrid
}

\begin{abstract}
Resumen: El propósito del presente estudio es determinar si una mecánica de tiro alternativa basada en la lateralidad óculo-manual del sujeto podría incrementar significativamente el porcentaje de acierto en el tiro a canasta en jugadores homogéneos - aquellos cuya lateralidad ocular y manual están en el mismo hemicuerpo lateral -. Para esto, 34 jugadores de baloncesto (24 chicos y 10 chicas) en categoría infantil, inscritos todos ellos en categoría Preferente masculino y femenino Federado, realizaron un total de 4600 lanzamientos a canasta desde cinco posiciones diferentes de corta y media distancia - 2300 con cada una de las dos mecánicas propuestas (mecánica tradicional y tiro con rotación de $45^{\circ}$ ) -. Tras el análisis de datos se hallaron porcentajes de acierto significativamente mayores desde el tiro libre $(\mathrm{t}(23)=$ 2,917; $\mathrm{p}=0,008)$, los lanzamientos desde el lado izquierdo de la zona (t(23) $=3,621 ; \mathrm{p}=0,001)$ y el global de los lanzamientos a canasta $(\mathrm{t}(23)=3,980$; $\mathrm{p}=0,001)$ con la mecánica de tiro teóricamente idónea. Los resultados obtenidos sustentan que una mecánica de tiro alternativa basada en la lateralidad óculo manual del jugador podría ayudar a los jugadores de baloncesto homogéneos a obtener porcentajes de acierto significativamente superiores. Palabras clave: Baloncesto - Tiro a Canasta - Dominancia Lateral.

Abstract: The purpose of the present study is to determine if a basketball shot mechanic based on the eye-hand laterality of the subject may produce a statistically significant increase in the average percentage in the basketball shot in homogeneous players - those whose hand and eye dominance are in the same body lateral -. To achieve this, 34 basketball players ( 24 boys and 10 girls) in U14 category, all of them registered in Preferente Masculino or Femenino Federado, made 4600 shots from 5 different positions of short and medium range -2300 shots with both mechanics proposed (traditional
\end{abstract}

mechanic and $45^{\circ}$ rotation shot) -. In the data analysis statistical significant differences were found in the free throw shots $(\mathrm{t}(23)=2,917 ; \mathrm{p}=0,008)$, the zone's left-side throws $(\mathrm{t}(23)=3,621 ; \mathrm{p} 0=0,001)$ and in the total of the basketball shots $(\mathrm{t}(23)=3,980 ; \mathrm{p}=0,001)$ with the shot mechanic theoretically ideal. The results obtained support that an alternative basketball shot mechanic based on the eye-hand laterality of the homogeneous players may help them to obtain average percentages significantly higher.

Key Words: Basketball - Basketball Shot - Lateral Dominance.

Resumo: o objetivo deste estudo é determinar se uma mecânica de lançamento alternativa com base na lateralidade oculo-manual incrementa significativamente a taxa de sucesso no lançamento de basquetbol em jogadores homógeneos. Neste estudo participaram, 34 jogadores de basquetebol (24 rapazes e 10 raparigas), do escaláo infantil, todos eles inscritos na liga de categoria Preferente masculino e Federado feminino, os atletas realizaron um total de 4600 lançamentos ao cesto de cinco posiçóes diferentes de curta e média distância - 2300 com cada um dos duas propostas mecânicas (mecânica tradicional e lançamento com rotação de $45^{\circ}$ ) -.Após a análise dos dados verificou-se porcentagens elevadas de sucesso nos lançamentos livres $(\mathrm{t}(23)=2.917, \mathrm{p}=0,008)$, nos lançamentos do lado esquerdo $(\mathrm{t}(23)=3.621 ; \mathrm{p}=0,001)$ e na totalidade dos lançamentos ao cesto $(\mathrm{t}(23)=$ 3.980; $\mathrm{p}=0,001)$ com a mecânica de lançamento teoricamente adecuada. Os resultados optidos sustentan que uma mecânica de lançamento com base na lateralidade oculo-manual do jogador poderia ajudar aos atletas de basquetebol homogenios a obter percentagens de sucesso no lançamento significativamente maiores.

Palavras-chave: Basquetebol - Lançamento ao Cesto - Dominancia Lateral.

\section{Introducción}

Diferentes publicaciones han señalado que el estudio de la lateralidad no debe limitarse exclusivamente a la preferencia manual (p.e.; Azemar y Massion, 2003; Del Valle y De la Vega, 2007). Para realizar éste de una forma completa se debe considerar también la lateralidad ocular, auditiva o podal, ya

\footnotetext{
Dirección para correspondencia [Correspondence address]: Carlos J. López Díaz. Facultad de Ciencias de la Actividad Física y el Deporte (INEF-UPM). cl Martín Fierro, 7. CP 28040, Madrid, Madrid (Espańa) E-mail: carlosjlopezd@gmail.com
}

que diferentes componentes corporales como los ojos, el oído o los pies cuentan, al igual que las manos, con su propia lateralidad y la interacción entre diferentes lateralidades - de forma más significativa las interacciones óculo-manual y óculo-podal - parecen condicionar el rendimiento deportivo de deportistas de diferentes disciplinas (p.e.; Azémar, Stein, y Ripoll, 2008; Dorochenko, 2009; Pluim, 2006).

En la lateralización ocular se podría definir un ojo no dominante y un ojo dominante o director. El ojo director es 
aquel que aporta la mayor parte de la información al cerebro y es el que aporta un mayor feedback, jugando un papel clave en ciertas técnicas deportivas (p.e.; Dorochenko, 2009; Pluim, 2006). Partiendo de esta idea, Dorochenko (2009), definió que cada sujeto podría ser clasificado en dos grandes grupos:

a. Jugadores homogéneos (HOM): aquellos cuya mano y ojo dominantes se encuentren en el mismo hemicuerpo.

b. Jugadores cruzados (CRU): aquellos cuya mano y ojo dominantes se encuentren en diferentes hemicuerpos.

Sillero, Refoyo, Lorenzo y Sampedro (2007), señalaron tras analizar las capacidades perceptivas visuales de jóvenes jugadores de baloncesto de alto nivel que los jugadores cruzados, pese a ser una minoría $(28,1 \%)$ presentan una prevalencia superior a lo normal en el mundo del baloncesto y podrían tener alguna posible ventaja de cara a un mayor rendimiento este deporte. Sin embargo, no definieron cuáles podrían ser estas ventajas, ni encontraron diferencias significativas en la percepción visual en función de la lateralidad óculo-manual.

$\mathrm{Si}$ se analizan diferentes gestos técnicos tipificados, se observa que el tiro a canasta podría ser una de las acciones condicionadas por la combinación de lateralidades ocular y manual. Son varios los estudios que analizan el porcentaje de acierto en la acción de tiro entendiéndolo como uno de los factores más determinantes en el resultado final de los partidos (p.e.; Ibáñez, García, Feu, Lorenzo y Sampaio, 2009; Ortega y Fernández, 2007; Sampaio y Leite, 2006; Cárdenas y Rojas, 1997; Gómez, Lorenzo, Ortega y Olmedilla, 2007; Fierro, 2002). Este gesto técnico ha sido estudiado tradicionalmente partiendo de una única mecánica de tiro basada en lanzar el balón con la mano dominante desde una posición inicial en la que se coloca el brazo de forma perpendicular al aro por encima del ojo (Patiño y López-Barrajón, 1998). Si se atiende a la clasificación propuesta por Dorochenko (2009) se comprende que esta mecánica podría suponer un claro hándicap a los jugadores homogéneos, dado que su ojo director se encuentra obstaculizado en el momento del tiro. En el trabajo de la mecánica de tiro, Claramunt y Balagué (2010), encontraron evidencias que sustentaban que se obtiene un menor porcentaje de acierto cuando los entrenadores fuerzan por medio del feedback verbal que el jugador se ajuste estrictamente al modelo tradicional (el cual podemos ver en la Figura 1).

Por otro lado, diversos estudios han destacado la importancia de la percepción visual en el tiro y son varios los autores que han señalado la clasificación del tiro en base a dos mecánicas de tiro diferentes en función de la localización del balón en relación a la línea ocular (p.e.; Oudejans, 2012; Stöckel y Breslin, 2013; Wu et al., 2013). Sin embargo, pese a que atendiendo a la clasificación de jugadores fundamentada en el principio de lateralidades múltiples explicada anterior- mente podría pensarse que la mecánica de tiro tradicional parece no ser la idónea para todos los tipos de jugadores; no han sido encontrados estudios que relacionen directamente el porcentaje de anotación con condicionantes en la lateralidad ocular del sujeto. Basándose en esta idea, podrían ser definidas dos mecánicas de tiro en función de la lateralidad óculo-manual del jugador:

a. Tiro con rotación de $45^{\circ}$; posiblemente el idóneo para jugadores homogéneos. Se basa en una modificación de la línea de los hombros, rotando ésta $45^{\circ}$ para liberar el ojo director en el momento del tiro.

b. Tiro tradicional o de frente; posiblemente el idóneo para jugadores cruzados. Es el que se ajusta al modelo tradicional de tiro, con la línea de los hombros en perpendicular al aro.

Figura 1. Ejemplo de posiciones de partida en ambas mecánicas.

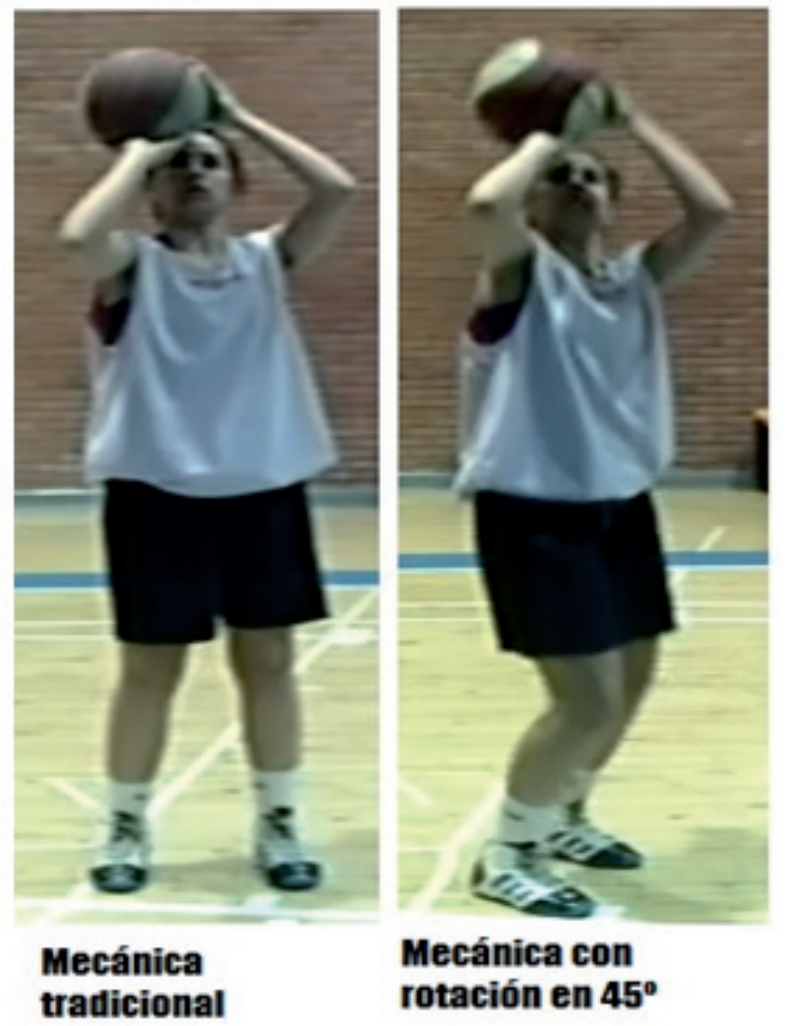

Por tanto, el objetivo principal del presente estudio es determinar si la aplicación de una mecánica de tiro alternativa, basada en el principio de lateralidades múltiples, podría incrementar significativamente el porcentaje de acierto en los lanzamientos a canasta de corta y media distancia frente a la aplicación exclusiva de una única mecánica de tiro tradicional en jugadores homogéneos.

Como objetivos secundarios, se pretende analizar, por un 
lado, si existen diferencias significativas en el porcentaje de acierto obtenido por los jugadores cruzados con cada una de las dos mecánicas; y por otro, se analizarán diferentes parámetros de capacidad visual en jugadores de baloncesto.

\section{Método}

El presente estudio se basa en una investigación de tipo preexperimental con un diseño pre- y post-tratamiento sobre la variable de eficiencia en el tiro a canasta utilizando el diseńo de doble ciego para evitar posibles sesgos en la información recogida (Thomas y Nelson, 2007).

En el presente estudio se analizaron 4600 lanzamientos a canasta, realizados por un total de 34 jugadores de baloncesto: 24 chicos pertenecientes a equipos inscritos en la liga Infantil Masculino Preferente A2 de la Federación de Baloncesto de Madrid (FBM), y 10 chicas participantes en la liga Infantil Femenino Federado División I de la FBM. La edad media de los participantes fue de 12,94 años (DT $=0,35$ ). Tanto los padres de los jugadores como los directores técnicos de los clubes participantes fueron informados con anterioridad de los propósitos de la investigación y las pruebas a realizar. Únicamente participaron en el estudio aquellos jugadores cuyos padres entregaron firmado el consentimiento informado antes de participar en el estudio.

Las mediciones llevadas a cabo fueron:

Pruebas de clasificación visual. - Con el objetivo de clasificar visualmente a los sujetos, se aplicaron el Test de Acercamiento Progresivo (TAP) para medir la agudeza visual binocular (AGVB) y monocular (AGVM) (Sillero y Bennett, 2001), y el Test de Desplazamiento Dorsal (TDD) para evaluar el campo visual periférico binocular (CVP) de los sujetos (Sillero, 1998). Ambas pruebas, siguiendo las recomendaciones de Sillero y Bennett (2000), fueron llevadas a cabo teniendo en cuenta la iluminación ambiente, desarrollándose en todos los casos dentro del rango fotópico (entre 100 y 3600 lux). Para la medición de la luz ambiente se utilizó un luxómetro corregido al seno, modelo Lunasix (Gossen, Alemania). Estas pruebas permitieron excluir de la muestra a aquellos jugadores cuya capacidad visual no alcanzaba el rango considerado como correcto (Sillero y Bennett, 2000)

Dominancia ocular. - Para la determinación del ojo director (OD) se pidió a los jugadores que mirasen a un punto fijo con ambos ojos abiertos a través de un hueco realizado con las dos manos unidas y ambos brazos extendidos; posteriormente se les pidió que acercasen progresivamente el hueco hacia el ojo que estuviese indicando el evaluador (Knudson y Kluka, 1997).Adicionalmente, también se aplicó el Test de Sighting (Dorochenko, 2009) basado en el test anterior pero, en lugar de mover las manos, se pidió a los jugadores que guińasen en primer lugar el ojo derecho y posteriormente el izquierdo y preguntándoles qué ojo no dejaba de ver el objetivo.
Ambos test se repitieron en dos ocasiones, si el OD coincidió en los cuatro casos no se repitió la prueba, en los casos en los que se obtuvieron resultados diferentes se repitió la prueba, hasta en tres ocasiones, considerándose el $\mathrm{OD}$ aquel que tuvo un mayor número de resultados positivos.

Dominancia manual. - Para la determinación de la lateralidad manual en el tiro se utilizó el Test de Harris (Rigal, 1992) consistente en llevar a cabo diferentes acciones tales como lanzar a canasta, sacar punta a un lapicero, cepillarse los dientes... con la mano con la que se realicen de forma más habitual.

Pruebas de efectividad en el tiro. - Una vez realizadas las pruebas de clasificación, se pidió a los jugadores que realizasen diez tiros a canasta con cada una de las mecánicas propuestas desde las posiciones 6, 7, 8, 9 y 10 del Campograma para el Análisis del Lanzamiento a Canasta de Ibáñez, Lozano y Martínez (2001). Las posiciones fueron delimitadas por un cono.

Figura 2. Campograma para el Análisis del Lanzamiento a Canasta (Ibáńez, Lozano y Martínez, 2001).

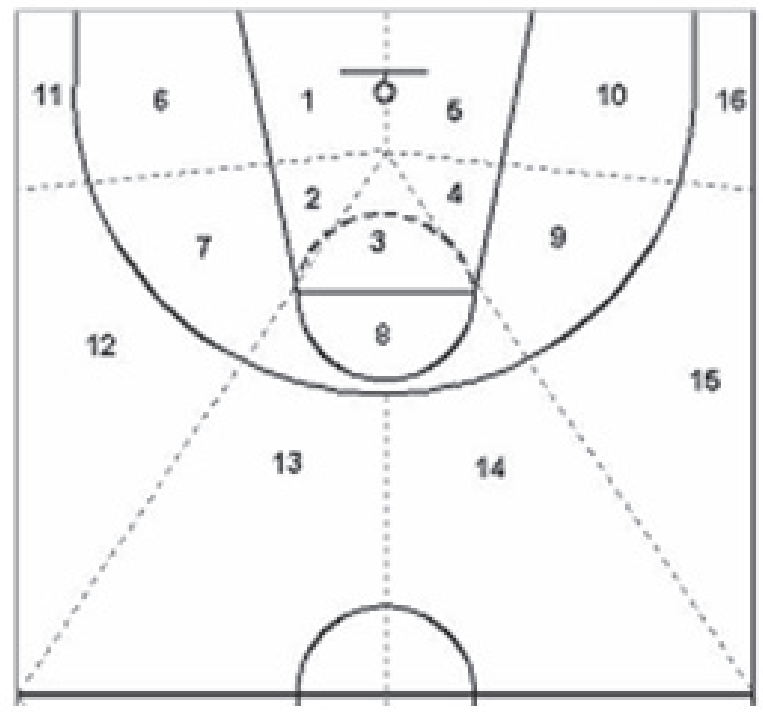

Un estudio piloto descartó las posiciones 1, 2, 3, 4 y 5 por ser posiciones muy cercanas al aro en las que la alta proporción de tiros encestados no permitiría ver las variaciones producidas en el porcentaje de anotación. Del mismo modo, las posiciones 11, 12, 13, 14, 15 y 16 fueron descartadas debido a que la distancia al aro obligaba a ciertos jugadores a modificar su mecánica natural para conseguir llegar al aro haciendo imposible la implantación de dos mecánicas diferentes y su posterior comparación.

El feedback dado a los jugadores se limitó a correcciones técnicas puntuales para garantizar que la mecánica utilizada era la que correspondía a la mecánica de tiro solicitada: el 
teóricamente idóneo (ID) o el teóricamente no idóneo (NID). Se hizo hincapié a los jugadores en la mayor importancia de una técnica correcta frente a la anotación o no del tiro. Todos los tiros fueron grabados con una cámara digital Samsung HMX-F90 colocada sobre un trípode bajo la canasta orientada de frente al sujeto para posteriormente comprobar que los tiros se hubiesen realizado de manera correcta. Se registró el resultado de cada tiro (anotado o fallado) con el fin de poder comparar los porcentajes de acierto totales y desde cada posición en el campo en función de la mecánica utilizada.

Para el análisis estadístico se utilizó el software IBM SPSS 20. Se estableció el límite de significación estadística en $\alpha$ $=0,05$ y todas aquellas correlaciones entre $\pm 0,75$ y \pm 1 . Para analizar la normalidad de las variables a estudiar se utilizaron las pruebas de Gráficos Q-Q y la Prueba de KolmogorovSmirnov. Una vez comprobados los criterios de normalidad se realizó una estadística descriptiva estableciendo los valores medios y desviaciones típicas para cada una de las variables en función de los diferentes grupos por variables independientes. Con el objetivo de comprobar si existía correlación entre diferentes variables se utilizó la prueba de Correlaciones bivariadas de Pearson. Se utilizó la Prueba T, tanto para muestras relacionadas como para muestras independientes asumiendo varianzas supuestamente iguales, one-way ANOVA, la prueba U de Mann-Whitney y la prueba de Chi cuadrado en función del tipo de variable considerado.

\section{Resultados}

Resultados de las habilidades visuales y la lateralidad.- Los datos de lateralidad óculo-manual apuntan que el 72,2\% de la muestra presentó una lateralidad homogénea y, el restante $27,3 \%$, cruzada. En la tabla 1 se recogen las preferencias en la lateralidad en función del grupo estudiado. No se encontraron diferencias significativas por género en la preferencia manual $(\mathrm{t}(31)=0,653 ; \mathrm{p}>0,05)$ ni en la ocular $(\mathrm{t}(31)=0,495$; $\mathrm{p}>0,05)$. Los datos recogidos sobre la combinación de ambas lateralidades tampoco sustentan la existencia de diferencias significativas por género $(\mathrm{t}(31)=0,225 ; \mathrm{p}>0,05)$.

Tabla 1. Estadísticos descriptivos de la lateralidad.

\begin{tabular}{lcc}
\hline & Izq (\%) & Drch (\%) \\
\hline OD & 24,2 & 75,8 \\
OD - Homogéneos & 0,0 & 100,0 \\
OD - Cruzados & 88,9 & 11,1 \\
MD & 3,0 & 97,0 \\
MD - Homogéneos & 0,0 & 100,0 \\
MD -Cruzados & 11,1 & 88,9
\end{tabular}

OD - Ojo dominante

MD - Mano dominante

En las tablas 2, 3 y 4 podemos observar los estadísticos descriptivos para la AV. Se observa que, pese a que la AV es ligeramente mayor en todos los valores para los sujetos homogéneos, los datos obtenidos no permiten afirmar que existan diferencias significativas para esta capacidad atendiendo a la lateralidad óculo-manual de los sujetos: ni para la AV binocular $(\mathrm{t}(31)=0,342 ; \mathrm{p}>0,05)$, el monocular del OD $(\mathrm{t}(31)=$ $0,181 ; \mathrm{p}>0,05)$ para el monocular del OD, o el monocular del No OD $(\mathrm{t}(31)=0,229 ; \mathrm{p}>0,05)$.

Tabla 2. Estadísticos descriptivos de la agudeza visual.

\begin{tabular}{lcccc}
\hline & Binocular & OD & No OD & Diferencia \\
\hline AV & $0,74 \pm 0,20$ & $0,67 \pm 0,21$ & $0,68 \pm 0,22$ & $0,01 \pm 0,08$ \\
AV Homogéneos & $0,75 \pm 0,21$ & $0,67 \pm 0,22$ & $0,69 \pm 0,23$ & $0,02 \pm 0,08$ \\
AV Cruzados & $0,72 \pm 0,19$ & $0,66 \pm 0,20$ & $0,67 \pm 0,21$ & $0,01 \pm 0,06$ \\
\hline \hline AV - Agudeza visual & & & & \\
OD - Ojo dominante & & & &
\end{tabular}

Tabla 3. Estadísticos de frecuencia de la capacidad visual.

\begin{tabular}{lccc}
\hline & Total (\%) & Homogéneos (\%) & Cruzados (\%) \\
\hline Deficiente & 27,3 & 25,0 & 33,3 \\
Correcta & 72,7 & 75,0 & 66,7 \\
Excelente & 0,0 & 0,0 & 0,0 \\
\hline Deficiente: $<0,66$ & & & \\
Correcta: $0,66-1,30$ & & & \\
Excelente: $>1,30$ & & &
\end{tabular}

\begin{tabular}{lccc}
\multicolumn{4}{l}{ Tabla 4. Estadísticos descriptivos del ojo de mayor agudeza visual. } \\
\hline & OD & no OD & Sin diferencia \\
\hline Total (\%) & 27,3 & 39,4 & 33,3 \\
Homogéneos (\%) & 25,0 & 29,2 & 45,8 \\
Cruzados (\%) & 33,3 & 66,7 & 0,0 \\
\hline \hline OD - Ojo dominante & &
\end{tabular}

En cuanto a la lateralidad del ojo de mayor AV, se observa un porcentaje ligeramente mayor de jugadores en los que su ojo no dominante muestra una mayor AV. Sin embargo, las 
pruebas comparativas realizadas no ponen de manifiesto la existencia de diferencias significativas en este apartado ni para el total de la población $(\mathrm{t}(32)=1,107$; $\mathrm{p}>0,05)$, ni para el análisis basado en la lateralidad óculo-manual: para sujetos homogéneos $(\mathrm{t}(23)=0,950 ; \mathrm{p}>0,05)$ o cruzados $(\mathrm{t}(8)=$ 0,553; p > 0,05).

Por último, el CVP medio total fue de $131,30 \pm 10,92^{\circ}$. Si analizamos éste atendiendo a la lateralidad óculo-manual de los sujetos, observamos que el CVP medio de los sujetos homogéneos $\left(131,38 \pm 11,34^{\circ}\right)$ es ligeramente mayor al de los cruzados $\left(131,11 \pm 10,36^{\circ}\right)$; sin embargo, la diferencia observada no permite afirmar que ésta sea significativa $(Z=0,066$; $\mathrm{p}>0,05)$.

Resultados de las pruebas en la mecánica de tiro.- Con respecto a la aplicación de una mecánica de tiro alternativa, los porcentajes de acierto obtenidos con cada una de las dos mecánicas de tiro a canasta se encuentran en la tabla 5 (Ver Tabla 5). Los resultados obtenidos sostienen que los jugadores homogéneos obtienen un porcentaje de acierto en el lanzamiento a canasta significativamente superior desde las posiciones $6(\mathrm{t}(23)=4,148 ; \mathrm{p}<0,001), 8(\mathrm{t}(23)=2,917 ; \mathrm{p}=0,008)$ y $10(\mathrm{t}(23)=2,373 ; \mathrm{p}=0,026)$; no habiendo sido encontradas diferencias significativas desde las posiciones $7(\mathrm{t}(23)=1,606$; $\mathrm{p}>0,05)$ y $9(\mathrm{t}(7)=0,882 ; \mathrm{p}>0,05)$. Estos datos reflejan que la mecánica de tiro con rotación de $45^{\circ}$ provoca una mejora significativa en el porcentaje de acierto en el tiro de estos jugadores desde el tiro libre $(\mathrm{t}(23)=2,917 ; \mathrm{p}=0,008)$, los tiros desde el lado izquierdo de la zona $(\mathrm{t}(23)=3,621 ; \mathrm{p}=0,001)$ y en el análisis global del total de los lanzamientos a canasta $(t(23)=3,980 ; \mathrm{p}=0,001)$.

En cuanto a los jugadores cruzados tan sólo muestran diferencias significativas en los porcentajes obtenidos desde la posición $7(\mathrm{t}(7)=4,583 ; \mathrm{p}=0,003)$; no habiendo encontrado diferencias significativas desde las posiciones $6(\mathrm{t}(7)=4,583$; $\mathrm{p}>0,05), 8(\mathrm{t} 7=2,007 ; \mathrm{p}>0,05), 9(\mathrm{t}(7)=0,882 ; \mathrm{p}>0,05) \mathrm{ni}$ $10(t(7)=1,528 ; \mathrm{p}>0,05)$. Pese a esto, el tratamiento global de los datos sí refleja un porcentaje de acierto significativamente mayor con la mecánica de tiro tradicional desde el lado derecho $(\mathrm{t}(7)=2,411 ; \mathrm{p}=0,047)$, así como en la totalidad de los lanzamientos $(\mathrm{t}(7)=2,897 ; \mathrm{p}=0,023)$.

En general, la población estudiada obtiene porcentajes de acierto significativamente superiores con su mecánica de tiro ID desde las posiciones $6(\mathrm{t}(31)=3,808 ; \mathrm{p}=0,001) ; 7(\mathrm{t}(31)$ $=3,056 ; \mathrm{p}=0,005) ; 8(\mathrm{t}(31)=3,590 ; \mathrm{p}=0,001)$ y $9(\mathrm{t}(31)=$ 1,044; $\mathrm{p}=0,305)$, no obteniéndose una mejora significativa desde la posición $10(\mathrm{t}(31)=2,871 ; \mathrm{p}>0,05)$.

Tabla 5. Porcentajes de acierto en el tiro a canasta antes del procedimiento.

\begin{tabular}{|c|c|c|c|c|}
\hline & & Tiro ID (\%) & Tiro NID (\%) & Diferencia (\%) \\
\hline \multirow{3}{*}{ Posición 6} & Total & $43,13 \pm 21,01$ & $27,19 \pm 12,50$ & $15,94 \pm 23,67^{*}$ \\
\hline & Homogéneos & $44,58 \pm 21,26$ & $25,42 \pm 13,18$ & $19,17 \pm 22,63^{*}$ \\
\hline & Cruzados & $38,75 \pm 21,00$ & $32,5 \pm 8,86$ & $6,25 \pm 25,60$ \\
\hline \multirow{3}{*}{ Posición 7} & Total & $37,5 \pm 16,66$ & $27,19 \pm 16,51$ & $10,31 \pm 19,09^{*}$ \\
\hline & Homogéneos & $35,83 \pm 18,16$ & $29,58 \pm 17,81$ & $6,25 \pm 19,07$ \\
\hline & Cruzados & $42,5 \pm 10,35$ & $20,0 \pm 9,26$ & $22,5 \pm 13,89^{*}$ \\
\hline \multirow{3}{*}{$\begin{array}{l}\text { Posición } 8 \\
\text { (Tiro Libre) }\end{array}$} & Total & $43,75 \pm 19,96$ & $28,13 \pm 15,95$ & $15,63 \pm 24,62^{*}$ \\
\hline & Homogéneos & $43,75 \pm 20,60$ & $29,17 \pm 17,17$ & $14,58 \pm 24,49^{*}$ \\
\hline & Cruzados & $43,75 \pm 19,23$ & $25,0 \pm 11,95$ & $18,75 \pm 26,42$ \\
\hline \multirow{3}{*}{ Posición 9} & Total & $31,88 \pm 15,33$ & $35,00 \pm 19,34$ & $3,13 \pm 16,93$ \\
\hline & Homogéneos & $30,0 \pm 15,88$ & $35,83 \pm 20,41$ & $5,83 \pm 16,66$ \\
\hline & Cruzados & $37,5 \pm 12,82$ & $32,5 \pm 16,69$ & $5,0 \pm 16,04$ \\
\hline \multirow{3}{*}{ Posición 10} & Total & $40,00 \pm 18,67$ & $29,38 \pm 14,58$ & $10,63 \pm 20,94^{*}$ \\
\hline & Homogéneos & $39,58 \pm 20,10$ & $29,58 \pm 13,67$ & $10,0 \pm 20,64^{*}$ \\
\hline & Cruzados & $41,25 \pm 14,58$ & $28,75 \pm 18,08$ & $12,5 \pm 23,15$ \\
\hline \multirow{3}{*}{ Total (6 a 10) } & Total & $39,25 \pm 12,87$ & $29,38 \pm 10,56$ & $9,88 \pm 11,29^{*}$ \\
\hline & Homogéneos & $38,75 \pm 14,07$ & $29,92 \pm 11,56$ & $8,83 \pm 10,87^{*}$ \\
\hline & Cruzados & $40,75 \pm 8,94$ & $27,75 \pm 7,13$ & $13,00 \pm 12,69^{*}$ \\
\hline \multirow{3}{*}{$\begin{array}{l}\text { Lado Drch } \\
(7 \text { y } 9)\end{array}$} & Total & $35,94 \pm 13,23$ & $32,19 \pm 13,50$ & $3,75 \pm 12,64$ \\
\hline & Homogéneos & $34,79 \pm 13,95$ & $32,71 \pm 14,29$ & $2,08 \pm 13,1$ \\
\hline & Cruzados & $39,38 \pm 10,84$ & $30,63 \pm 11,48$ & $8,75 \pm 10,26^{*}$ \\
\hline
\end{tabular}




\begin{tabular}{llccc}
\hline & & Tiro ID (\%) & Tiro NID (\%) & Diferencia (\%) \\
\hline \multirow{2}{*}{ Lado Izq } & Total & $40,31 \pm 15,18$ & $27,19 \pm 12,18$ & $13,13 \pm 16,60^{*}$ \\
$(6$ y 10) & Homogéneos & $40,21 \pm 16,78$ & $27,5 \pm 13,35$ & $12,71 \pm 17,19^{*}$ \\
& Cruzados & $40,63 \pm 9,80$ & $26,25 \pm 8,35$ & $14,38 \pm 15,68^{*}$ \\
\hline
\end{tabular}

Tiro ID - Tiro teóricamente idóneo

Tiro NID - Tiro teóricamente no idóneo

TL - Tiro libre

Lado Drch - Tiros efectuados desde el lado derecho de la zona

Lado Izq - Tiros efectuados desde el lado izquierdo de la zona

*Diferencia significativa al $95 \%$

\section{Discusión}

En cuanto al objeto principal de estudio, los resultados obtenidos muestran que, la aplicación de una mecánica de tiro alternativa basada en el principio de lateralidades múltiples, presenta un incremento significativo del porcentaje de acierto en los lanzamientos a canasta de corta y media distancia en jugadores homogéneos. Podría afirmarse, por tanto, que la mecánica de tiro tradicional explicada por Patiño y LópezBarrajón (1998), no es la idónea para la totalidad de los sujetos (Claramunt y Balagué, 2010), ya que no se ajusta a las características individuales inherentes a cada individuo, particularmente con la lateralidad óculo-manual.

En definitiva, las datos recogidos ponen de manifiesto que la mecánica de tiro alternativa (tiro con rotación de 45\%) permitiría a los jugadores homogéneos obtener porcentajes de acierto significativamente mayores en los lanzamientos a canasta de corta y media distancia; recordemos que los lanzamientos de larga distancia no han sido analizados debido a características de los sujetos que no lo hacían viable. Se hace por tanto necesario un estudio llevado a cabo con jugadores cuyas características permitan comprobar esta idea en lanzamientos de larga distancia. Este hecho sustenta la idea defendida por Dorochenko (2009), de que adaptaciones técnico-tácticas basadas en el principio de lateralidades múltiples permiten mejorar significativamente el rendimiento del deportista. Debe recordarse que esta es la primera aplicación de una mecánica de tiro basada en el principio de lateralidades múltiples de la que tenemos constancia en baloncesto, pero dicho principio ya ha sido aplicado exitosamente por el mismo autor en deportes como el tenis o el fútbol (Dorochenko, 2013).

Así mismo, se corrobora la importancia de liberar el ojo director en el lanzamiento con el objetivo de mejorar la capacidad visual, aspecto que ya había sido destacado por diferentes estudios que comprobaron la importancia de la capacidad visual en la eficacia en el lanzamiento a canasta (p.e.; Oudejans, 2012; Stöckel y Breslin, 2013; Wu, et al., 2013). Por otra parte, se comprueba que la combinación de las diferentes lateralidades del sujeto puede ser un elemento de gran valía a la hora de planificar el entrenamiento de los sujetos de cara a su rendimiento deportivo (Pluim, 2006).
En cuanto a los objetivos secundarios de estudio, el análisis de datos realizado pone de manifiesto que la mecánica de tiro tradicional permite a los sujetos con lateralidad cruzada obtener mejores porcentajes de acierto que el tiro en 45․ Esto podría deberse a una mayor eficiencia de la mecánica de tiro explicada por Patiño y López-Barrajón (1998), y al hecho de que su capacidad visual no se vea mermada por las propias características de la mecánica de tiro, hecho que sí se produce en los jugadores homogéneos y que sustenta el desarrollo de una mecánica de tiro alternativa. En definitiva, tal y como señalan Sillero et al. (2007), la mecánica de tiro tradicional supone una ventaja competitiva para los jugadores cruzados.

En lo referente a las pruebas de clasificación visual, si bien es cierto que las diferencias obtenidas parecen no condicionar el porcentaje de acierto en el tiro a canasta, al comparar las características de esta muestra con la utilizada por Sillero et al. (2007), se destacan dos aspectos: a) que la capacidad visual de sujetos de categoría preferente de la FBM es inferior a la de jugadores de su misma edad en selección nacional: basándonos en esto, convendría realizar un estudio con una mayor muestra para comprobar si el tener una mayor capacidad visual pudiera ser un indicador de éxito o una variable discriminante a aplicar en los procesos de detección de talentos y b) la homogeneidad de la muestra utilizada en el presente estudio podría ser óbice para la inexistencia de dichas diferencias, por lo que convendría realizar un estudio que compare el porcentaje de acierto en el lanzamiento a canasta con la capacidad visual utilizando una muestra más heterogénea.

\section{Aplicaciones Prácticas}

Se recomienda incluir es el estudio del principio de lateralidades múltiples en los planes de formación de entrenadores de baloncesto, así como las dos mecánicas de tiro propuestas en función de la lateralidad óculo-manual del sujeto.

En cuanto a los entrenadores, se les recomienda evaluar la lateralidad óculo-manual de sus jugadores con el fin de poder entrenarlos en la mecánica de tiro a canasta que se ajuste en mayor medida a sus necesidades. 
Agradecimientos.- Queríamos darle las gracias a los tres clubes participantes, Club Baloncesto Femenino Alcorcón, Club Baloncesto Getafe Beta y Club Baloncesto Pozuelo; así como a sus jugadores y entrenadores Álex, Dani y Ramiro, ya que sin ellos este estudio no habría podido ser llevado a cabo. Muchas gracias.

\section{Referencias}

1. Azemar, G., y Massion, J. (2003). L’homme asymétrique. CNRS Editions: París. ISBN: 9782271061799.

2. Azemar, G., Stein, J.F., y Ripoll, H. (2008). Effects of ocular dominance on eye-hand coordination in sporting duels. Science \& Sports, 23 (6), 263-277-

3. Cárdenas, D., y Rojas, J. (1997). Determinación de la incidencia del tiro libre en el resultado final a través del análisis estadístico. Revista Motricidad, 3, 177-186.

4. Claramunt Aguayo, C., y Balagué Serre, N. (2010). Influencia de las instrucciones técnicas en la efectividad del tiro en baloncesto. Apunts. Educación Física y Deportes, 99, 65-71.

5. Del Valle Díaz, S., y De la Vega Marcos, R. (2007). Lateralidad en el deporte de full contact. Cambios en diferentes condiciones. Revista Internacional de Medicina y Ciencias de la Actividad Física y el Deporte, 25 (7), 32-51.

6. Dorochenko, P. (2009). Interés de las lateralidades en el deporte. Paper presented at the XXII Jornades de medicina de l'ésport del Bages, Manresa. Recuperado de http://www.jmebages.cat/pdf/CR2.pdf (Encontrado el 29/10/2013).

7. Dorochenko, P. (2013). El Ojo Director [Versión para lector digital]. Paul Dorochenko. Recuperado de http://www.amazon.es/El-OjoDirector-Paul-Dorochenko-ebook/dp/B00E41NVGG (Encontrado el 28/05/2014).

8. Fierro Hernández, C. (2002). Variables relacionadas con el éxito deportivo en las ligas NBA y ACB de baloncesto. Revista de Psicología del Deporte, 11(2), 247-255.

9. Gómez, M.A., Lorenzo, A., Ortega, E., y Olmedilla, A. (2007). Diferencias de los indicadores de rendimiento en baloncesto femenino entre ganadores y perdedores en función de jugar como local o como visitante. Revista de Piscología del Deporte, 16(1), 41-54.

10. Hageman, N. (2009). The advantage of being left-handed in interactive sports. Attention, Perception, \& Psychophysics, 71(7), 1641-1648.

11. Ibáñez, S. J., García, J., Feu, S., Lorenzo, A., y Sampaio, J. (2009). Effects of consecutive basketball games in the game-related statistics that discriminate winner and losing games teams. Journal of Sports Science and Medicine, 8, 458-462.

12. Ibáńez, S.J., Lozano, A., y Martínez, B. (2001). Análisis del tiro a canasta en función del tipo y valor de los lanzamientos, género y nivel de los jugadores. En J. García, S.J. Ibáñez, S. Feu, M. Cañadas y I. Parejo. Estudio de la gestoforma del lanzamiento a canasta en liga E.B.A. Retos. Nuevas tendencias en Educación Física, Deporte y Recreación.
13. Knudson, D., y Kluka, D. A. (1997). The impact of visión and visión training on sport performance. Journal of Health, Physical Education, Recreation and Dance, 68(4), 17-24.

14. Ortega, E., y Fernández, R. (2007). Diferencias en el lanzamiento de 3 puntos entre ganadores y perdedores en etapas de formación. Paper presented at the Congreso Ibérico de Baloncesto, Cáceres.

15. Oudejans, R. R. D. (2012). Effects of visual control training on the shooting performance of elite female basketball players. International Journal of Sports Science \& Coaching, 7(3), 469-480.

16. Patiño Resa, A., y López-Barrajón Sevilla, M. (1998). Aplicaciones del análisis y evaluación de la técnica en baloncesto. Apunts. Educación Fisica y Deportes, 54, 37-43.

17. Pluim, B. (2006). ¿Consideraciones médicas en la detección de talentos? ITF, Coaching \& Sport Science Review, 14(39), 6.

18. Rigal, R.A. (1992). Whick handedness: preference of performance? Perceptual and Motor Skills, 75, 851-866.

19. Sampaio, J., y Leite, N. (2006). ¿¿Por qué ganaron o perdieron los partidos de baloncesto los equipos que participaron en el Eurobasket 2005? Kronos, 9, 67-73.

20. Sillero Quintana, M., Refoyo Román, I., Lorenzo Calvo, A., y Sampedro Molinuevo, J. (2007). Perceptual visual skills in young highly skilled basketball players. Perceptual and Motor Skills, 104, 547-561.

21. Sillero, M. (1998). Comparación de técnicas para evaluación de la Agudeza Visual y los extremos del campo visual horizontal. Archivos Optométricos, 2 (2), 86-96.

22. Sillero, M., y Bennett, S. (2000). Proceso de validación del test de Acercamiento Progresivo bajo cuatro condiciones de iluminación ambiente. Actas del I Congreso de la Asociación Española de Ciencias del Deporte (I), Cáceres, Espańa.

23. Sillero, M., y Bennett, S. (2001). Validation of the Bringing Nearer Test for visual acuity testing using different background light conditions. Proceedings of the 6th Annual Congress of the European College of Sport Science. Julio, 2001, Colonia, Alemania.

24. Stöckel, T., y Breslin, G. (2013). The influence of visual contextual information on the emergence of the especial skill in basketball. Journal of Sport \& Exercise Psychology, 35, 536-541.

25. Thomas, J.R., y Nelson, J.K. (2007). Métodos de investigación en actividad física. Editorial Paidotribo: Barcelona. ISBN: 9788480198707.

26. Wu, Y., Zeng, Y., Zhang, L., Wang, S., Wang, D., Tan, X., Zhu, X., Zhang, J., y Zhang, J. (2013). The role of visual perception in action anticipation in basketball athletes. Neuroscience, 237, 29-41. 